\title{
First Observation of the Radiative Decay $\Lambda_{b}^{0} \rightarrow \Lambda \gamma$
}

\author{
R. Aaij et al. \\ (LHCb Collaboration)
}

(Received 23 April 2019; published 15 July 2019)

\begin{abstract}
The radiative decay $\Lambda_{b}^{0} \rightarrow \Lambda \gamma$ is observed for the first time using a data sample of proton-proton collisions corresponding to an integrated luminosity of $1.7 \mathrm{fb}^{-1}$ collected by the LHCb experiment at a center-of-mass energy of $13 \mathrm{TeV}$. Its branching fraction is measured exploiting the $B^{0} \rightarrow K^{* 0} \gamma$ decay as a normalization mode and is found to be $\mathcal{B}\left(\Lambda_{b}^{0} \rightarrow \Lambda \gamma\right)=(7.1 \pm 1.5 \pm 0.6 \pm 0.7) \times 10^{-6}$, where the quoted uncertainties are statistical, systematic, and systematic from external inputs, respectively. This is the first observation of a radiative decay of a beauty baryon.
\end{abstract}

DOI: 10.1103/PhysRevLett.123.031801

The decay $\Lambda_{b}^{0} \rightarrow \Lambda \gamma$ proceeds via the $b \rightarrow s \gamma$ flavorchanging neutral-current transition. This process is forbidden at tree level in the standard model (SM) and is, therefore, sensitive to new particles entering the loop-level transition, which can modify decay properties. The polarization of the photon in these processes is predicted to be predominantly left-handed in the SM, up to small corrections of the order $m_{s} / m_{b}$ [1]. While precise measurements of branching fractions and charge-parity-violation observables in radiative $b$-meson decays previously performed at the BABAR, Belle, and LHCb collaborations [2-5] are in agreement with SM calculations [6-12], they do not provide stringent constraints on the presence of righthanded contributions to $b \rightarrow s$ gamma transitions [1316]. Radiative $b$-baryon decays have never been observed and offer a unique benchmark for measuring the photon polarization due to the nonzero spin of the initial- and finalstate particles [17]. In particular, the $\Lambda_{b}^{0} \rightarrow \Lambda \gamma$ decay has been proposed as a suitable mode for the study of the photon polarization, since the helicity of the $\Lambda$ baryon can be measured, giving access to the helicity structure of the $b \rightarrow s \gamma$ transition $[18,19]$.

The $\Lambda_{b}^{0} \rightarrow \Lambda \gamma$ decay is experimentally challenging to reconstruct. At high-energy hadron colliders, the $\Lambda_{b}^{0}$ decay vertex cannot be determined directly due to the long lifetime of the weakly decaying $\Lambda$ baryon and the unknown photon direction, when reconstructed as a cluster in the electromagnetic calorimeter. Photons converting to a pair of electrons in the detector material could be used to reconstruct the photon direction but at the cost of a large

*Full author list given at the end of the article.

Published by the American Physical Society under the terms of the Creative Commons Attribution 4.0 International license. Further distribution of this work must maintain attribution to the author(s) and the published article's title, journal citation, and DOI. Funded by SCOAP ${ }^{3}$. efficiency loss. This approach was used by the CDF experiment to set the best limit on the branching fraction of this decay, $\mathcal{B}\left(\Lambda_{b}^{0} \rightarrow \Lambda \gamma\right)<1.3 \times 10^{-3}$ at $90 \%$ C.L. [20]. This measurement still leaves ample room for improvement before achieving a sensitivity comparable to the SM prediction of $\mathcal{B}\left(\Lambda_{b}^{0} \rightarrow \Lambda \gamma\right)$, which lies in the range $(6-500) \times 10^{-7}$, where the large variation is due to different computations of the $\Lambda_{b}^{0} \rightarrow \Lambda$ form factors at the photon pole [21-27]. A precise measurement of the branching fraction of this decay allows discrimination between different approaches to the form-factor computation and is an important step towards the measurement of the photon polarization in radiative $b$-baryon decays.

The $\mathrm{LHCb}$ experiment provides unique conditions for studying the $\Lambda_{b}^{0} \rightarrow \Lambda \gamma$ mode thanks to the large production of $\Lambda_{b}^{0}$ baryons at the LHC [28,29] and the excellent properties of the detector optimized for the analysis of $b$ -hadron decays. This Letter presents the first observation of the $\Lambda_{b}^{0} \rightarrow \Lambda \gamma$ decay, with $\Lambda$ reconstructed as $\Lambda \rightarrow p \pi^{-}$, by the $\mathrm{LHCb}$ experiment. The well-known radiative decay $B^{0} \rightarrow K^{* 0} \gamma$ [30] is used as a normalization mode to measure the $\Lambda_{b}^{0} \rightarrow \Lambda \gamma$ branching fraction. The data sample used in this Letter corresponds to $1.7 \mathrm{fb}^{-1}$ of integrated luminosity collected by the LHCb experiment in $13 \mathrm{TeV}$ proton-proton $(p p)$ collisions during 2016. The results were not inspected until all analysis procedures were finalized.

The $\mathrm{LHCb}$ detector $[31,32]$ is a single-arm forward spectrometer covering the pseudorapidity range $2<\eta<5$. The detector includes a high-precision tracking system consisting of a silicon-strip vertex detector surrounding the $p p$ interaction region, a large-area silicon-strip detector located upstream of a dipole magnet with a bending power of about $4 \mathrm{Tm}$, and three stations of silicon-strip detectors and straw drift tubes placed downstream of the magnet. The tracking system provides a measurement of the momentum, $p$, of charged particles with a relative uncertainty that varies 
from $0.5 \%$ at low momentum to $1.0 \%$ at $200 \mathrm{GeV}$. (Natural units with $\hbar=c=1$ are used throughout, so that mass and momentum are measured in units of energy.) The minimum distance of a track to a primary vertex (PV), is measured with a resolution of $\left(15+29 / p_{T}\right) \mu \mathrm{m}$, where $p_{T}$ is the component of the momentum transverse to the beam, in $\mathrm{GeV}$. Different types of charged hadrons are distinguished using information from two ring-imaging Cherenkov detectors. Photons, electrons, and hadrons are identified by a calorimeter system consisting of scintillating-pad and preshower detectors, an electromagnetic, and a hadronic calorimeter. Charged and neutral clusters in the electromagnetic calorimeter are separated by extrapolating the tracks reconstructed by the tracking system to the calorimeter plane, while photons and neutral pions are distinguished by cluster shape and energy distributions. For decays with high-energy photons in the final state, such as $B^{0} \rightarrow K^{* 0} \gamma$, a $B^{0}$ mass resolution around $100 \mathrm{MeV}$ is achieved $[16,33]$, dominated by the photon energy resolution. The online event selection is performed by a trigger, which consists of a hardware stage, based on information from the calorimeter and muon systems, followed by a software stage, which applies a full event reconstruction.

At the hardware-trigger stage, events are required to have a cluster in the electromagnetic calorimeter with transverse energy $E_{T}$ above a threshold that varies in the range 2.1-3.0 GeV. The software trigger requires at least one charged particle to have transverse momentum $p_{T}>1 \mathrm{GeV}$ and to be inconsistent with originating from any PV. Finally, a vertex is formed with two tracks significantly displaced from any $\mathrm{PV}$ and the combination with a high- $E_{T}$ photon is used to identify decays consistent with the signal and normalization modes. In the off-line selection, trigger signals are associated with reconstructed particles. Only events in which the trigger was fired due to the signal candidate are kept.

Simulated events are used to model the effects of the detector acceptance and the imposed selection requirements. In the simulation, $p p$ collisions are generated using PYTHIA [34] with a specific LHCb configuration [35]. Decays of unstable particles are described by EVTGEN [36], in which final-state radiation is generated using PHOTOS [37]. The interaction of the generated particles with the detector and its response are implemented using the GEANT4 toolkit [38] as described in Ref. [39]. The signal sample is generated with unpolarized $\Lambda_{b}^{0}$ and only a lefthanded photon contribution. The agreement between data and simulation is validated using the $\Lambda_{b}^{0} \rightarrow J / \psi p K^{-}$, $\Lambda_{b}^{0} \rightarrow J / \psi \Lambda$, and $B^{0} \rightarrow K^{* 0} \gamma$ control modes exploiting the selections described in Refs. [40,41], and [16], respectively. The $\Lambda_{b}^{0}$ momentum distribution of all simulated samples involving $\Lambda_{b}^{0}$ decays is corrected for discrepancies between the data and simulation in two-dimensional bins of $\Lambda_{b}^{0}$ momentum and $p_{T}, p\left(\Lambda_{b}^{0}\right)$, and $p_{T}\left(\Lambda_{b}^{0}\right)$, using
$\Lambda_{b}^{0} \rightarrow J / \psi p K^{-}$background-subtracted data and simulated candidates.

Signal candidates are reconstructed from the combination of a $\Lambda$ baryon and a high-energy photon candidate. Good-quality tracks, consistent with the proton and pion hypotheses, with opposite charge and well separated from any PV, are combined to form the $\Lambda$ candidate. Proton and pion candidates are required to have $p_{T}$ larger than 800 and $300 \mathrm{MeV}$, respectively. The proton-pion system is required to have an invariant mass in the range of 1110-1122 MeV and to form a good vertex that is well separated from the nearest PV. Only $\Lambda$ candidates that decay in the highly segmented part of the vertex detector $(z<270 \mathrm{~mm})$ and have a $p_{T}$ larger than $1 \mathrm{GeV}$ are retained for further study. Photons, reconstructed from clusters in the electromagnetic calorimeter, must be consistent with those originating from a neutral particle and have $E_{T}>3 \mathrm{GeV}$. The photon direction is computed assuming it is produced in the interaction region. The sum of the $\Lambda p_{T}$ and the photon $E_{T}$ should be larger than $5 \mathrm{GeV}$. The $\Lambda_{b}^{0}$ four-momentum is obtained as the sum of the $\Lambda$ and photon candidate fourmomenta. The $\Lambda_{b}^{0}$ transverse momentum is required to be above $4 \mathrm{GeV}$ and its invariant mass within $900 \mathrm{MeV}$ of the known $\Lambda_{b}^{0}$ mass [42]. Since the origin vertex of the photon is not known, the $\Lambda_{b}^{0}$ decay vertex is not reconstructed, and therefore, it is not possible to use its displacement with respect to the PV to separate background coming directly from the $p p$ collision. Instead, the distance of closest approach (DOCA) between the $\Lambda_{b}^{0}$ and $\Lambda$ trajectories is required to be small, where the former is calculated using the reconstructed momentum and assuming it originates at the PV closest to the $\Lambda$ trajectory. Candidates for the normalization channel $B^{0} \rightarrow K^{* 0} \gamma$ are reconstructed following similar criteria. In this case, tracks are required to be consistent with the $K$ and $\pi$ hypotheses, their invariant mass must be within $100 \mathrm{MeV}$ of the known $K^{* 0}$ mass [42], and the $B^{0}$ candidate mass is required to be in the range of 4600-6180 MeV.

A boosted decision tree (BDT) [43], employing the XGBoost algorithm [44] and implemented through the sCIKIT-learn library [45], is used to further separate signal from combinatorial background. It is trained on simulated events as proxy to the signal and on data candidates with an invariant mass larger than $6.1 \mathrm{GeV}$ as background. A combination of topological and isolation information is used as input for the classifier, including the transverse momentum and the separation from the PV of the different particles, the separation between the $\Lambda$ decay vertex and the PV and the DOCA between the two tracks and between the $\Lambda_{b}^{0}$ and $\Lambda$ trajectories. Background $\Lambda_{b}^{0}$ candidates with extra tracks close to the $\Lambda$ or photon candidates are rejected using the asymmetry of the sum of momenta of all the tracks present in a cone of $1 \mathrm{rad}$ around the particle direction with respect to its momentum. Such tracks potentially arise from 
decays with additional particles in the final state that have not been reconstructed when building the $\Lambda_{b}^{0}$ candidate. A twofold technique [46] is used to avoid overtraining and no correlation is observed between the BDT response and the candidate mass. The requirement on the BDT output is optimized using the Punzi figure of merit [47]. The chosen working point provides a background rejection of $99.8 \%$ while retaining $33 \%$ of the signal candidates. A separate BDT with the same configuration and input variables is trained to select $B^{0} \rightarrow K^{* 0} \gamma$ candidates using simulated candidates as signal and data events in the high-mass sideband as background. In this case, the requirement on the BDT output is optimized by maximizing the signal significance using the known branching fraction for this decay to compute the expected signal yield at each step.

Potential contamination from neutral pions that are reconstructed as a single merged cluster in the electromagnetic calorimeter is suppressed by employing a neural network classifier trained to separate $\pi^{0}$ mesons from photons. This classifier exploits the broader shape of the calorimeter cluster of a $\pi^{0}$ meson with respect to that of a single photon by using as input a set of variables based on the combination of shower shape and energy information from the different calorimeter subsystems [48].

The invariant-mass distribution of the selected candidates is used to disentangle signal from background through a maximum likelihood fit. The $\Lambda_{b}^{0} \rightarrow \Lambda \gamma$ signal component is modeled with a double-tailed Crystal Ball [49] probability density function (PDF), with power-law tails above and below the $\Lambda_{b}^{0}$ mass. The tail parameters are fixed to values determined from simulation while the mean and width of the signal peak are related to those of the $B^{0}$ meson using simulation and the mass difference between the $\Lambda_{b}^{0}$ and $B^{0}$ hadrons measured by LHCb [50]. Several sources of background are investigated, but only two are found to be significant. The narrow width of the $\Lambda$ baryon [42] and the clean signature of the high- $p_{T}$ proton allow a pure hadronic selection, reducing the contamination from charged particle misidentification, e.g., coming from $K_{\mathrm{S}}^{0} \rightarrow \pi^{+} \pi^{-}$decays misidentified as $\Lambda \rightarrow p \pi^{-}$candidates, to a negligible level. Potentially dangerous backgrounds from decays with a similar topology to the signal and an additional pion have been studied and found to be negligible. Decays with intermediate $\Lambda_{c}^{+}$states, like $\Lambda_{b}^{0} \rightarrow$ $\Lambda_{c}^{+} \pi^{-}$with $\Lambda_{c}^{+} \rightarrow \Lambda \pi^{+} \pi^{0}$, are found to populate an invariant-mass range outside our fit region, and the topologically similar decay $\Lambda_{b}^{0} \rightarrow \Lambda \pi^{0}$ is expected to be suppressed due to the absence of QCD penguin contributions in this decay mode [51]. The dominant source of background is formed by combinations of a real $\Lambda$ baryon with a random photon, referred to as combinatorial background, and is modeled with an exponential PDF with a free decay parameter. A small contamination from $\Lambda_{b}^{0} \rightarrow \Lambda \eta$ decays with $\eta \rightarrow \gamma \gamma$, where one of the photons is not reconstructed, is also expected and is described with the shape determined from simulation. The signal and combinatorial yields are free to float in the fit to data, while the yield of $\Lambda_{b}^{0} \rightarrow \Lambda \eta$ is constrained using the known branching fraction [42] and the reconstruction and selection efficiencies determined from simulation.

The mass distribution of $B^{0} \rightarrow K^{* 0} \gamma$ signal candidates is also described by a Crystal Ball function with two powerlaw tails with the parameters obtained from simulated events. The combinatorial component is modeled as an exponential PDF. Partially reconstructed backgrounds, i.e., background decays where one or more particles have not been reconstructed, are copious in this case, mostly originating from the charged meson $B^{+}$. Three contributions are accounted for and modeled with shapes obtained from simulation: two inclusive ones encompassing decays where one pion has not been reconstructed, referred to as $B \rightarrow K^{+} \pi^{-} \pi \gamma$, and decays with a neutral pion in the final state and any missing particle, referred to as $B \rightarrow K^{+} \pi^{-} \pi^{0} X$; and $B^{0} \rightarrow K^{* 0} \eta$ decays, where one of the photons from the $\eta \rightarrow \gamma \gamma$ decay has not been reconstructed. Backgrounds due to particle misidentification are also more abundant in this case, due to the broad width of the $K^{* 0}$ meson [42]. Contributions from $B_{s}^{0} \rightarrow \phi \gamma, \Lambda_{b}^{0} \rightarrow p K^{-} \gamma$, and $B^{0} \rightarrow K^{+} \pi^{-} \pi^{0}$ decays are described with the shapes obtained from simulation. The yields of the signal, combinatorial, and inclusive partially reconstructed background are allowed to float in the fit, while those of the $B^{0} \rightarrow K^{* 0} \eta$, $B_{s}^{0} \rightarrow \phi \gamma, \Lambda_{b}^{0} \rightarrow p K^{-} \gamma$, and $B^{0} \rightarrow K^{+} \pi^{-} \pi^{0}$ decays are fixed to the values obtained from simulation and the measured branching fractions [42,52]. The fit stability is validated by performing pseudoexperiments with various signal yield hypotheses before proceeding with the final fit to data. It is also checked that the extraction of the signal branching fraction is unbiased for branching fraction hypotheses at least as large as $3 \times 10^{-6}$.

The yield of signal and normalization events is obtained from a simultaneous extended unbinned maximum likelihood fit to data. The ratio of yields is given by the expression

$$
\begin{aligned}
\frac{N\left(\Lambda_{b}^{0} \rightarrow \Lambda \gamma\right)}{N\left(B^{0} \rightarrow K^{* 0} \gamma\right)}= & \frac{f_{\Lambda_{b}^{0}}}{f_{B^{0}}} \times \frac{\mathcal{B}\left(\Lambda_{b}^{0} \rightarrow \Lambda \gamma\right)}{\mathcal{B}\left(B^{0} \rightarrow K^{* 0} \gamma\right)} \times \frac{\mathcal{B}\left(\Lambda \rightarrow p \pi^{-}\right)}{\mathcal{B}\left(K^{* 0} \rightarrow K^{+} \pi^{-}\right)} \\
& \times \frac{\epsilon\left(\Lambda_{b}^{0} \rightarrow \Lambda \gamma\right)}{\epsilon\left(B^{0} \rightarrow K^{* 0} \gamma\right)},
\end{aligned}
$$

where $f_{\Lambda_{b}^{0}} / f_{B^{0}}$ is the ratio of hadronization fractions, $\mathcal{B}$ is the branching fraction and $\epsilon$ is the combined reconstruction and selection efficiency for the given decay. The latter is obtained from simulation, except for the efficiencies related to charged particle identification requirements, which are determined from calibration samples of $\Lambda \rightarrow p \pi^{-}$ and $D^{0} \rightarrow K^{-} \pi^{+}$[53]. The results of the simultaneous fit to data candidates are shown in Fig. 1. The signal yields 

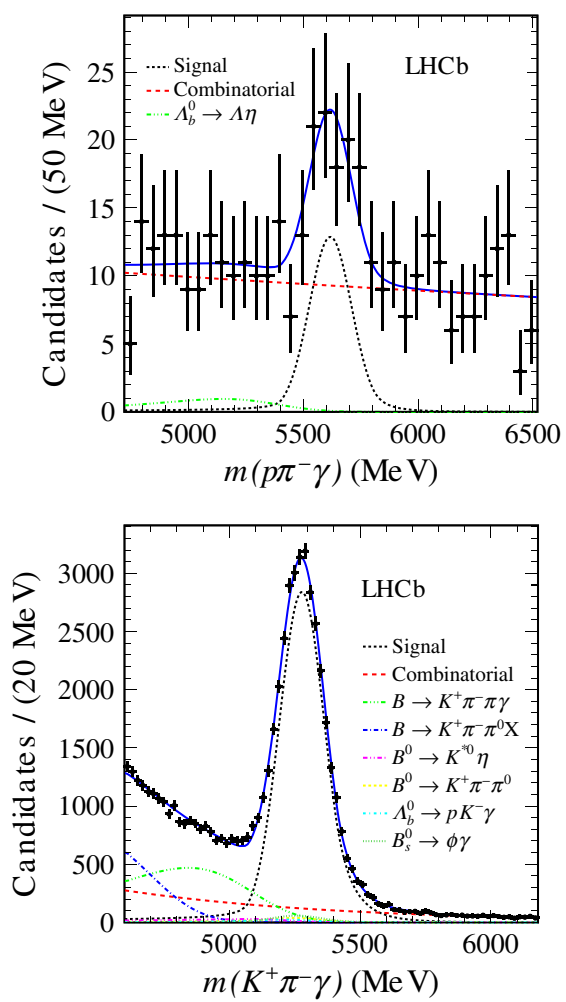

FIG. 1. Simultaneous fit to the (top) $\Lambda_{b}^{0} \rightarrow \Lambda \gamma$ and (bottom) $B^{0} \rightarrow K^{* 0} \gamma$ invariant-mass distributions of selected candidates. The data are represented by black dots and the result of the fit by a solid blue curve while individual contributions are represented in different line styles (see legend).

are found to be $65 \pm 13$ and $32670 \pm 290$ for $\Lambda_{b}^{0} \rightarrow \Lambda \gamma$ and $B^{0} \rightarrow K^{* 0} \gamma$, respectively. The ratio of hadronization and branching fractions is measured to be

$$
\begin{aligned}
& \frac{f_{\Lambda_{b}^{0}}}{f_{B^{0}}} \times \frac{\mathcal{B}\left(\Lambda_{b}^{0} \rightarrow \Lambda \gamma\right)}{\mathcal{B}\left(B^{0} \rightarrow K^{* 0} \gamma\right)} \times \frac{\mathcal{B}\left(\Lambda \rightarrow p \pi^{-}\right)}{\mathcal{B}\left(K^{* 0} \rightarrow K^{+} \pi^{-}\right)} \\
& \quad=(9.9 \pm 2.0) \times 10^{-2},
\end{aligned}
$$

where the uncertainty is statistical only. To determine the signal branching fraction, the ratio of hadronization fractions, $f_{\Lambda_{b}^{0}} / f_{B^{0}}$, is computed from the LHCb measurement of this quantity as a function of the $p_{T}$ of the $b$ baryon [29] and from the distribution of $p_{T}\left(\Lambda_{b}^{0}\right)$ in the signal simulation. An average over $p_{T}$ of the ratio of hadronization fractions of $f_{\Lambda_{b}^{0}} / f_{B^{0}}=0.60 \pm 0.05$ is obtained for this analysis, where the uncertainty is derived from Ref. [29]. Taking the known branching fractions of the normalization mode and intermediate decays from Ref. [42], the signal branching fraction is measured to be

$$
\mathcal{B}\left(\Lambda_{b}^{0} \rightarrow \Lambda \gamma\right)=(7.1 \pm 1.5) \times 10^{-6},
$$

where the uncertainty is statistical only.
TABLE I. Dominant systematic uncertainties on the measurement of $\mathcal{B}\left(\Lambda_{b}^{0} \rightarrow \Lambda \gamma\right)$. The uncertainties arising from external measurements are given separately.

\begin{tabular}{lc}
\hline \hline Source & Uncertainty $(\%)$ \\
\hline Data/simulation agreement & 7.7 \\
$\Lambda_{b}^{0}$ fit model & 3.0 \\
$B^{0} \rightarrow K^{* 0} \gamma$ backgrounds & 2.7 \\
Size of simulated samples & 1.7 \\
Efficiency ratio & 1.4 \\
\hline Sum in quadrature & 9.0 \\
\hline$f_{\Lambda_{b}^{0}} / f_{B^{0}}$ & 8.7 \\
Input branching fractions & 3.0 \\
\hline Sum in quadrature & 9.2 \\
\hline \hline
\end{tabular}

Using the sPlot [54] technique, the absence of potential remaining backgrounds entering in the signal component is cross-checked. In particular, the invariant mass of the $p \pi$ system and the output of the neural network classifier separating $\pi^{0}$ mesons from photons for backgroundsubtracted data candidates are found to be compatible with the expected signal distributions.

The dominant systematic uncertainties are listed in Table I. The largest contribution arises from the limited knowledge of the ratio of hadronization fractions, $f_{\Lambda_{b}^{0}} / f_{B^{0}}$. Potential remaining differences between data and simulation are evaluated by changing the requirement on the BDT output, recomputing the efficiencies, and repeating the mass fit. Further systematic uncertainties come from the limited precision of the input branching fractions, the signal and normalization fit models, the finite simulation samples used to compute the selection efficiencies, and other uncertainties associated to the extraction of the ratio of efficiencies, including the uncertainties on the corrections applied to the simulation and systematic effects on the extraction of the particle identification and hardware trigger efficiencies.

The $\Lambda_{b}^{0} \rightarrow \Lambda \gamma$ signal significance is evaluated from a profile likelihood using Wilks' theorem [55] and is confirmed with pseudoexperiments. Including both statistical and systematic uncertainties, the $\Lambda_{b}^{0} \rightarrow \Lambda \gamma$ decay is observed with a significance of $5.6 \sigma$.

To summarize, a search for the $b$-baryon flavorchanging neutral-current radiative decay $\Lambda_{b}^{0} \rightarrow \Lambda \gamma$ is performed with a data sample corresponding to an integrated luminosity of $1.7 \mathrm{fb}^{-1}$ collected in $p p$ collisions at a center-of-mass energy of $13 \mathrm{TeV}$ with the LHCb detector. A signal of $65 \pm 13$ decays is observed with a significance of $5.6 \sigma$. This is the first observation of this mode and represents the first step towards the study of the photon polarization in radiative decays of $b$-baryons with a larger dataset. Exploiting the well-known $B^{0} \rightarrow K^{* 0} \gamma$ mode as a 
normalization channel, the branching fraction of the $\Lambda_{b}^{0} \rightarrow \Lambda \gamma$ decay is measured for the first time, $\mathcal{B}\left(\Lambda_{b}^{0} \rightarrow \Lambda \gamma\right)=$ $(7.1 \pm 1.5 \pm 0.6 \pm 0.7) \times 10^{-6}$, where the first uncertainty is statistical, the second systematic, and the third is the systematic from external measurements. Our result is in good agreement with the predictions from Refs. [22], [23] and [27], which make use of light cone sum rules, the heavy quark limit and the covariant constituent quark model, respectively. A more recent calculation [26], which relies on the relativistic quark model and is able to predict accurately the integrated $\mathcal{B}\left(\Lambda_{b}^{0} \rightarrow \Lambda \mu^{+} \mu^{-}\right)$measured by LHCb [56], is compatible with the rate of $\Lambda_{b}^{0} \rightarrow \Lambda \gamma$, although no uncertainties on this calculation are available. Other predictions [21,24,25] are further away from our result, which can be used as input to future revisions.

We express our gratitude to our colleagues in the CERN accelerator departments for the excellent performance of the LHC. We thank the technical and administrative staff at the LHCb institutes. We acknowledge support from CERN and from the national agencies: CAPES, CNPq, FAPERJ, and FINEP (Brazil); MOST and NSFC (China); CNRS/ IN2P3 (France); BMBF, DFG, and MPG (Germany); INFN (Italy); NWO (Netherlands); MNiSW and NCN (Poland); MEN/IFA (Romania); MSHE (Russia); MinECo (Spain); SNSF and SER (Switzerland); NASU (Ukraine); STFC (United Kingdom); NSF (USA). We acknowledge the computing resources that are provided by CERN, IN2P3 (France), KIT and DESY (Germany), INFN (Italy), SURF (Netherlands), PIC (Spain), GridPP (United Kingdom), RRCKI and Yandex LLC (Russia), CSCS (Switzerland), IFIN-HH (Romania), CBPF (Brazil), PL-GRID (Poland), and OSC (USA). We are indebted to the communities behind the multiple open-source software packages on which we depend. Individual groups or members have received support from AvH Foundation (Germany); EPLANET, Marie Skłodowska-Curie Actions, and ERC (European Union); ANR, Labex P2IO and OCEVU, and Région Auvergne-Rhône-Alpes (France); Key Research Program of Frontier Sciences of CAS, CAS PIFI, and the Thousand Talents Program (China); RFBR, RSF, and Yandex LLC (Russia); GVA, XuntaGal, and GENCAT (Spain); the Royal Society and the Leverhulme Trust (United Kingdom); Laboratory Directed Research and Development program of LANL (USA).

[1] D. Atwood, M. Gronau, and A. Soni, Mixing Induced $C P$ Asymmetries in Radiative $B$ Decays in and beyond the Standard Model, Phys. Rev. Lett. 79, 185 (1997).

[2] J. P. Lees et al. (BABAR Collaboration), Precision Measurement of the $B \rightarrow X_{s} \gamma$ Photon Energy Spectrum, Branching Fraction, and Direct $C P$ Asymmetry $A_{C P}\left(B \rightarrow X_{s+d} \gamma\right)$, Phys. Rev. Lett. 109, 191801 (2012).
[3] T. Saito et al. (Belle Collaboration), Measurement of the $\bar{B} \rightarrow X_{s} \gamma$ branching fraction with a sum of exclusive decays, Phys. Rev. D 91, 052004 (2015).

[4] T. Horiguchi et al. (Belle Collaboration), Evidence for Isospin Violation and Measurement of $C P$ Asymmetries in $B \rightarrow K^{*}(892) \gamma$, Phys. Rev. Lett. 119, 191802 (2017).

[5] R. Aaij et al. (LHCb Collaboration), Measurement of the ratio of branching fractions $\mathcal{B}\left(B^{0} \rightarrow K^{* 0} \gamma\right) / \mathcal{B}\left(B_{s}^{0} \rightarrow \phi \gamma\right)$ and the direct $C P$ asymmetry in $B^{0} \rightarrow K^{* 0} \gamma$, Nucl. Phys. B867, 1 (2013).

[6] S. W. Bosch and G. Buchalla, The radiative decays $B \rightarrow V \gamma$ at next-to-leading order in QCD, Nucl. Phys. B621, 459 (2002).

[7] M. Beneke, Th. Feldmann, and D. Seidel, Exclusive radiative and electroweak $b \rightarrow d$ and $b \rightarrow s$ penguin decays at NLO, Eur. Phys. J. C 41, 173 (2005).

[8] B. Grinstein and D. Pirjol, Long distance effects in $B \rightarrow V \gamma$ radiative weak decays, Phys. Rev. D 62, 093002 (2000).

[9] M. Matsumori, A. I. Sanda, and Y. Y. Keum, $C P$ asymmetry, branching ratios and isospin breaking effects of $B \rightarrow K^{*} \gamma$ with perturbative QCD approach, Phys. Rev. D 72, 014013 (2005).

[10] P. Ball, G. W. Jones, and R. Zwicky, $B \rightarrow V \gamma$ beyond QCD factorization, Phys. Rev. D 75, 054004 (2007).

[11] M. Misiak, H. M. Asatrian, R. Boughezal, M. Czakon, T. Ewerth, A. Ferroglia, P. Fiedler, P. Gambino, C. Greub, U. Haisch et al., Updated NNLO QCD Predictions for the Weak Radiative B-Meson Decays, Phys. Rev. Lett. 114, 221801 (2015).

[12] A. Paul and D. M. Straub, Constraints on new physics from radiative $B$ decays, J. High Energy Phys. 04 (2017) 027.

[13] Y. Ushiroda et al. (Belle Collaboration), Time-dependent $C P$ asymmetries in $B^{0} \rightarrow K_{S}^{0} \pi^{0} \gamma$ transitions, Phys. Rev. D 74, 111104 (2006).

[14] B. Aubert et al. (BABAR Collaboration), Measurement of time-dependent $C P$ asymmetry in $B^{0} \rightarrow K_{S}^{0} \pi^{0} \gamma$ decays, Phys. Rev. D 78, 071102 (2008).

[15] R. Aaij et al. (LHCb Collaboration), Angular analysis of the $B^{0} \rightarrow K^{* 0} e^{+} e^{-}$decay in the low- $q^{2}$ region, J. High Energy Phys. 04 (2015) 064.

[16] R. Aaij et al. (LHCb Collaboration), First Experimental Study of Photon Polarization in Radiative $B_{s}^{0}$ Decays, Phys. Rev. Lett. 118, 021801 (2017).

[17] M. Gremm, F. Krüger, and L. M. Sehgal, Angular distribution and polarization of photons in the inclusive decay $\Lambda_{b}^{0} \rightarrow X_{s} \gamma$, Phys. Lett. B 355, 579 (1995).

[18] T. Mannel and S. Recksiegel, Flavour-changing neutral current decays of heavy baryons. The case $\Lambda_{b}^{0} \rightarrow \Lambda \gamma$, J. Phys. G 24, 979 (1998).

[19] G. Hiller and A. Kagan, Probing for new physics in polarized $\Lambda_{b}^{0}$ decays at the $Z$ pole, Phys. Rev. D 65, 074038 (2002).

[20] D. Acosta et al. (CDF Collaboration), Search for radiative $b$-hadron decays in $p \bar{p}$ collisions at $\sqrt{s}=1.8 \mathrm{TeV}$, Phys. Rev. D 66, 112002 (2002).

[21] H.-Y. Cheng, C.-Y. Cheung, G.-L. Lin, Y. C. Lin, T.-M. Yan, and H.-L. Yu, Effective Lagrangian approach to weak radiative decays of heavy hadrons, Phys. Rev. D 51, 1199 (1995). 
[22] Y.-M. Wang, Y. Li, and C.-D. Lü, Rare decays of $\Lambda_{b}^{0} \rightarrow \Lambda \gamma$ and $\Lambda_{b}^{0} \rightarrow \Lambda \ell^{+} \ell^{-}$in the light-cone sum rules, Eur. Phys. J. C 59, 861 (2009).

[23] T. Mannel and Y.-M. Wang, Heavy-to-light baryonic form factors at large recoil, J. High Energy Phys. 12 (2011) 067.

[24] L.-F. Gan, Y.-L. Liu, W.-B. Chen, and M.-Q. Huang, Improved light-cone QCD sum rule analysis of the rare decays $\Lambda_{b}^{0} \rightarrow \Lambda \gamma$ and $\Lambda_{b}^{0} \rightarrow \Lambda l^{+} l^{-}$, Commun. Theor. Phys. 58, 872 (2012).

[25] K. Azizi, S. Kartal, A. T. Olgun, and Z. Tavukoglu, Analysis of the radiative $\Lambda_{b}^{0} \rightarrow \Lambda \gamma$ transition in standard model and scenarios with one or two universal extra dimensions, Phys. Rev. D 88, 015030 (2013).

[26] R. N. Faustov and V. O. Galkin, Rare $\Lambda_{b}^{0} \rightarrow \Lambda l^{+} l^{-}$and $\Lambda_{b}^{0} \rightarrow \Lambda \gamma$ decays in the relativistic quark model, Phys. Rev. D 96, 053006 (2017).

[27] T. Gutsche, M. A. Ivanov, J. G. Korner, V. E. Lyubovitskij, and P. Santorelli, Rare baryon decays $\Lambda_{b} \rightarrow \Lambda l^{+} l^{-}(l=$ $e, \mu, \tau)$ and $\Lambda_{b} \rightarrow \Lambda \gamma$ : differential and total rates, leptonand hadron-side forward-backward asymmetries, Phys. Rev. D 87, 074031 (2013).

[28] R. Aaij et al. (LHCb Collaboration), Measurement of the $b$-Quark Production Cross-Section in 7 and $13 \mathrm{TeV} p p$ Collisions, Phys. Rev. Lett. 118, 052002 (2017); Erratum, Phys. Rev. Lett. 119, 169901(E) (2017).

[29] R. Aaij et al. (LHCb Collaboration), Measurement of $b$-Hadron Fractions in $13 \mathrm{TeV} p p$ Collisions, arXiv: 1902.06794 [Phys. Rev. Lett. (to be published)].

[30] Y. Amhis et al. (Heavy Flavor Averaging Group), Averages of $b$-hadron, $c$-hadron, and $\tau$-lepton properties as of summer 2016, Eur. Phys. J. C 77, 895 (2017), updated results and plots available at https://hflav.web.cern.ch.

[31] A. A. Alves Jr. et al. (LHCb Collaboration), The LHCb detector at the LHC, J. Instrum. 3, S08005 (2008).

[32] R. Aaij et al. (LHCb Collaboration), LHCb detector performance, Int. J. Mod. Phys. A 30, 1530022 (2015).

[33] R. Aaij et al. (LHCb Collaboration), Measurement of the ratio of branching fractions $\mathcal{B}\left(B^{0} \rightarrow K^{* 0} \gamma\right) / \mathcal{B}\left(B_{s}^{0} \rightarrow \phi \gamma\right)$, Phys. Rev. D 85, 112013 (2012).

[34] T. Sjöstrand, S. Mrenna, and P. Skands, A brief introduction to PYTHIA 8.1, Comput. Phys. Commun. 178, 852 (2008); T. Sjöstrand, S. Mrenna, and P. Skands, PYTHIA 6.4 physics and manual, J. High Energy Phys. 05 (2006) 026.

[35] I. Belyaev et al., Handling of the generation of primary events in Gauss, the LHCb simulation framework, J. Phys. Conf. Ser. 331, 032047 (2011).

[36] D. J. Lange, The EvTGEN particle decay simulation package, Nucl. Instrum. Methods Phys. Res., Sect. A 462, 152 (2001).

[37] P. Golonka and Z. Was, Рнотоs Monte Carlo: A precision tool for QED corrections in $Z$ and $W$ decays, Eur. Phys. J. C 45, 97 (2006).

[38] J. Allison et al. (GEANT4 Collaboration), GEANT4 developments and applications, IEEE Trans. Nucl. Sci. 53 (2006) 270; S. Agostinelli et al. (GEANT4 Collaboration), GEANT4: A simulation toolkit, Nucl. Instrum. Methods Phys. Res., Sect. A 506, 250 (2003).
[39] M. Clemencic, G. Corti, S. Easo, C. R. Jones, S. Miglioranzi, M. Pappagallo, and P. Robbe, The LHCb simulation application, Gauss: Design, evolution and experience, J. Phys. Conf. Ser. 331, 032023 (2011).

[40] R. Aaij et al. (LHCb Collaboration), Observation of the suppressed decay $\Lambda_{b}^{0} \rightarrow p \pi^{-} \mu^{+} \mu^{-}$, J. High Energy Phys. 04 (2017) 029.

[41] R. Aaij et al. (LHCb Collaboration), Measurements of the $\Lambda_{b}^{0} \rightarrow J / \psi \Lambda$ decay amplitudes and the $\Lambda_{b}^{0}$ polarisation in $p p$ collisions at $\sqrt{s}=7 \mathrm{TeV}$, Phys. Lett. B 724, 27 (2013).

[42] M. Tanabashi et al. (Particle Data Group), Review of particle physics, Phys. Rev. D 98, 030001 (2018).

[43] L. Breiman, J. H. Friedman, R. A. Olshen, and C. J. Stone, Classification and regression trees (Wadsworth International Group, Belmont, CA, 1984).

[44] T. Chen and C. Guestrin, XGBoost: A scalable tree boosting system, in Proceedings of the 22Nd ACM SIGKDD International Conference on Knowledge Discovery and Data Mining, KDD '16, (ACM, New York, 2016), p. 785, http://doi.acm.org/10.1145/2939672.2939785.

[45] F. Pedregosa et al., ScIKIT-learn: Machine learning in Python, J. Machine Learning Res. 12, 2825 (2011).

[46] A. Blum et al., Beating the hold-out: Bounds for $k$-fold and progressive cross-validation, in Proceedings of the Twelfth Annual Conference on Computational Learning Theory, in COLT (ACM, New York, 1999), p. 203, https://doi.org/ 10.1145/307400.307439.

[47] G. Punzi, Sensitivity of searches for new signals and its optimization, eConf C030908, MODT002 (2003).

[48] M. Calvo Gomez et al., A tool for $\gamma / \pi^{0}$ separation at high energies, CERN Report No. LHCb-PUB-2015-016, 2015.

[49] T. Skwarnicki, A study of the radiative cascade transitions between the Upsilon-prime and Upsilon resonances, Ph.D. thesis, Institute of Nuclear Physics, 1986; CERN Report No. DESY-F31-86-02, 1990.

[50] R. Aaij et al. (LHCb Collaboration), Study of Beauty Hadron Decays into Pairs of Charm Hadrons, Phys. Rev. Lett. 112, 202001 (2014).

[51] J. Zhu, Z.-T. Wei, and H.-W. Ke, Semileptonic and nonleptonic weak decays of $\Lambda_{b}^{0}$, Phys. Rev. D 99, 054020 (2019).

[52] V. J. Rives Molina, Study of $b$-hadron decays into two hadrons and a photon at $\mathrm{LHCb}$ and first observation of $b$ baryon radiative decays, Ph.D. thesis, Universitat de Barcelona, 2016.

[53] R. Aaij et al., Selection and processing of calibration samples to measure the particle identification performance of the LHCb experiment in Run 2, EPJ Tech. Instrum. 6, 1 (2019).

[54] M. Pivk and F. R. Le Diberder, sPlot: A statistical tool to unfold data distributions, Nucl. Instrum. Methods Phys. Res., Sect. A 555, 356 (2005).

[55] S. S. Wilks, The large-sample distribution of the likelihood ratio for testing composite hypotheses, Ann. Math. Stat. 9, 60 (1938).

[56] R. Aaij et al. (LHCb Collaboration), Differential branching fraction and angular analysis of $\Lambda_{b}^{0} \rightarrow \Lambda \mu^{+} \mu^{-}$decays, J. High Energy Phys. 06 (2015) 115; Erratum, J. High Energy Phys. 09 (2018) 145(E). 
R. Aaij, ${ }^{29}$ C. Abellán Beteta, ${ }^{46}$ B. Adeva, ${ }^{43}$ M. Adinolfi, ${ }^{50}$ C. A. Aidala,${ }^{77}$ Z. Ajaltouni, ${ }^{7}$ S. Akar, ${ }^{61}$ P. Albicocco, ${ }^{20}$ J. Albrecht, ${ }^{12}$ F. Alessio, ${ }^{44}$ M. Alexander, ${ }^{55}$ A. Alfonso Albero, ${ }^{42}$ G. Alkhazov,${ }^{35}$ P. Alvarez Cartelle, ${ }^{57}$ A. A. Alves Jr., ${ }^{43}$

S. Amato, ${ }^{2}$ Y. Amhis, ${ }^{9}$ L. An, ${ }^{19}$ L. Anderlini, ${ }^{19}$ G. Andreassi, ${ }^{45}$ M. Andreotti, ${ }^{18}$ J. E. Andrews, ${ }^{62}$ F. Archilli, ${ }^{29}$

J. Arnau Romeu, ${ }^{8}$ A. Artamonov, ${ }^{41}$ M. Artuso,${ }^{63}$ K. Arzymatov, ${ }^{39}$ E. Aslanides, ${ }^{8}$ M. Atzeni,${ }^{46}$ B. Audurier,${ }^{24}$ S. Bachmann, ${ }^{14}$ J. J. Back, ${ }^{52}$ S. Baker, ${ }^{57}$ V. Balagura, ${ }^{9, b}$ W. Baldini, ${ }^{18,44}$ A. Baranov, ${ }^{39}$ R. J. Barlow, ${ }^{58}$ S. Barsuk, ${ }^{9}$ W. Barter ${ }^{57}$ M. Bartolini, ${ }^{21}$ F. Baryshnikov, ${ }^{73}$ V. Batozskaya, ${ }^{33}$ B. Batsukh, ${ }^{63}$ A. Battig, ${ }^{12}$ V. Battista, ${ }^{45}$ A. Bay, ${ }^{45}$ F. Bedeschi, ${ }^{26}$ I. Bediaga, ${ }^{1}$ A. Beiter, ${ }^{63}$ L. J. Bel, ${ }^{29}$ S. Belin,${ }^{24}$ N. Beliy, ${ }^{4}$ V. Bellee, ${ }^{45}$ N. Belloli, ${ }^{22, c}$ K. Belous,${ }^{41}$ I. Belyaev,${ }^{36}$ G. Bencivenni, ${ }^{20}$ E. Ben-Haim, ${ }^{10}$ S. Benson ${ }^{29}$ S. Beranek, ${ }^{11}$ A. Berezhnoy, ${ }^{37}$ R. Bernet, ${ }^{46}$ D. Berninghoff, ${ }^{14}$ E. Bertholet,${ }^{10}$ A. Bertolin, ${ }^{25}$ C. Betancourt, ${ }^{46}$ F. Betti,${ }^{17, d}$ M. O. Bettler ${ }^{51}$ Ia. Bezshyiko, ${ }^{46}$ S. Bhasin, ${ }^{50}$ J. Bhom,${ }^{31}$ M. S. Bieker, ${ }^{12}$ S. Bifani, ${ }^{49}$ P. Billoir, ${ }^{10}$ A. Birnkraut, ${ }^{12}$ A. Bizzeti, ${ }^{19, \mathrm{e}}$ M. Bjørn,${ }^{59}$ M. P. Blago, ${ }^{44}$ T. Blake, ${ }^{52}$ F. Blanc, ${ }^{45}$ S. Blusk ${ }^{63}$ D. Bobulska, ${ }^{55}$ V. Bocci, ${ }^{28}$ O. Boente Garcia, ${ }^{43}$ T. Boettcher, ${ }^{60}$ A. Bondar, ${ }^{40, f}$ N. Bondar, ${ }^{35}$ S. Borghi, ${ }^{58,44}$ M. Borisyak,${ }^{39}$ M. Borsato, ${ }^{14}$ M. Boubdir, ${ }^{11}$ T. J. V. Bowcock,${ }^{56}$ C. Bozzi,${ }^{18,44}$ S. Braun, ${ }^{14}$ M. Brodski, ${ }^{44}$ J. Brodzicka, ${ }^{31}$

A. Brossa Gonzalo, ${ }^{52}$ D. Brundu, ${ }^{24,44}$ E. Buchanan, ${ }^{50}$ A. Buonaura, ${ }^{46}$ C. Burr, ${ }^{58}$ A. Bursche, ${ }^{24}$ J. S. Butter, ${ }^{29}$ J. Buytaert ${ }^{44}$ W. Byczynski, ${ }^{44}$ S. Cadeddu, ${ }^{24}$ H. Cai, ${ }^{67}$ R. Calabrese, ${ }^{18, g}$ S. Cali, ${ }^{20}$ R. Calladine, ${ }^{49}$ M. Calvi, ${ }^{22, c}$ M. Calvo Gomez, ${ }^{42, h}$ A. Camboni, ${ }^{42, \mathrm{~h}}$ P. Campana, ${ }^{20}$ D. H. Campora Perez,${ }^{44}$ L. Capriotti, ${ }^{17, \mathrm{~d}}$ A. Carbone, ${ }^{17, \mathrm{~d}}$ G. Carboni, ${ }^{27}$ R. Cardinale, ${ }^{21}$ A. Cardini, ${ }^{24}$ P. Carniti, ${ }^{22, c}$ K. Carvalho Akiba, ${ }^{2}$ G. Casse,${ }^{56}$ M. Cattaneo, ${ }^{44}$ G. Cavallero, ${ }^{21}$ R. Cenci, ${ }^{26, i}$ M. G. Chapman,${ }^{50}$ M. Charles, ${ }^{10,44} \mathrm{Ph}$. Charpentier, ${ }^{44}$ G. Chatzikonstantinidis, ${ }^{49}$ M. Chefdeville, ${ }^{6}$ V. Chekalina, ${ }^{39}$ C. Chen, ${ }^{3}$ S. Chen, ${ }^{24}$ S.-G. Chitic,${ }^{44}$ V. Chobanova, ${ }^{43}$ M. Chrzaszcz, ${ }^{44}$ A. Chubykin, ${ }^{35}$ P. Ciambrone,${ }^{20}$ X. Cid Vidal, ${ }^{43}$ G. Ciezarek, ${ }^{44}$ F. Cindolo, ${ }^{17}$ P. E. L. Clarke, ${ }^{54}$ M. Clemencic, ${ }^{44}$ H. V. Cliff, ${ }^{51}$ J. Closier, ${ }^{44}$ V. Coco, ${ }^{44}$ J. A. B. Coelho, ${ }^{9}$ J. Cogan, ${ }^{8}$ E. Cogneras, ${ }^{7}$ L. Cojocariu, ${ }^{34}$ P. Collins,${ }^{44}$ T. Colombo, ${ }^{44}$ A. Comerma-Montells, ${ }^{14}$ A. Contu, ${ }^{24}$ G. Coombs, ${ }^{44}$

S. Coquereau, ${ }^{42}$ G. Corti, ${ }^{44}$ C. M. Costa Sobral,${ }^{52}$ B. Couturier, ${ }^{44}$ G. A. Cowan,${ }^{54}$ D. C. Craik,${ }^{60}$ A. Crocombe,${ }^{52}$ M. Cruz Torres, ${ }^{1}$ R. Currie, ${ }^{54}$ C. L. Da Silva, ${ }^{78}$ E. Dall'Occo, ${ }^{29}$ J. Dalseno,${ }^{43, j}$ C. D'Ambrosio, ${ }^{44}$ A. Danilina,${ }^{36}$ P. d'Argent, ${ }^{14}$ A. Davis ${ }^{58}$ O. De Aguiar Francisco, ${ }^{44}$ K. De Bruyn, ${ }^{44}$ S. De Capua ${ }^{58}$ M. De Cian, ${ }^{45}$ J. M. De Miranda,${ }^{1}$ L. De Paula, ${ }^{2}$ M. De Serio, ${ }^{16, k}$ P. De Simone, ${ }^{20}$ J. A. de Vries,${ }^{29}$ C. T. Dean,${ }^{55}$ W. Dean ${ }^{77}$ D. Decamp,${ }^{6}$ L. Del Buono, ${ }^{10}$ B. Delaney, ${ }^{51}$ H.-P. Dembinski, ${ }^{13}$ M. Demmer, ${ }^{12}$ A. Dendek,${ }^{32}$ D. Derkach,${ }^{74}$ O. Deschamps, ${ }^{7}$ F. Desse, ${ }^{9}$ F. Dettori, ${ }^{24}$ B. Dey, ${ }^{68}$ A. Di Canto, ${ }^{44}$ P. Di Nezza, ${ }^{20}$ S. Didenko, ${ }^{73}$ H. Dijkstra, ${ }^{44}$ F. Dordei, ${ }^{24}$ M. Dorigo, ${ }^{26,1}$ A. C. dos Reis, ${ }^{1}$ A. Dosil Suárez, ${ }^{43}$ L. Douglas, ${ }^{55}$ A. Dovbnya,${ }^{47}$ K. Dreimanis,${ }^{56}$ L. Dufour,${ }^{44}$ G. Dujany, ${ }^{10}$ P. Durante, ${ }^{44}$ J. M. Durham,${ }^{78}$ D. Dutta, ${ }^{58}$ R. Dzhelyadin, ${ }^{41, a}$ M. Dziewiecki, ${ }^{14}$ A. Dziurda, ${ }^{31}$ A. Dzyuba ${ }^{35}$ S. Easo ${ }^{53}$ U. Egede ${ }^{57}$ V. Egorychev,${ }^{36}$ S. Eidelman,${ }^{40, f}$ S. Eisenhardt ${ }^{54}$ U. Eitschberger, ${ }^{12}$ R. Ekelhof, ${ }^{12}$ L. Eklund, ${ }^{55}$ S. Ely, ${ }^{63}$ A. Ene, ${ }^{34}$ S. Escher, ${ }^{11}$ S. Esen, ${ }^{29}$ T. Evans, ${ }^{61}$ A. Falabella ${ }^{17}$ C. Färber, ${ }^{44}$ N. Farley, ${ }^{49}$ S. Farry, ${ }^{56}$ D. Fazzini, ${ }^{22, c}$ M. Féo, ${ }^{44}$ P. Fernandez Declara, ${ }^{44}$ A. Fernandez Prieto, ${ }^{43}$ F. Ferrari, ${ }^{17, d}$ L. Ferreira Lopes ${ }^{45}$ F. Ferreira Rodrigues, ${ }^{2}$ S. Ferreres Sole, ${ }^{29}$ M. Ferro-Luzzi, ${ }^{44}$ S. Filippov,${ }^{38}$ R. A. Fini, ${ }^{16}$ M. Fiorini, ${ }^{18, \mathrm{~g}}$ M. Firlej, ${ }^{32}$ C. Fitzpatrick, ${ }^{44}$ T. Fiutowski, ${ }^{32}$ F. Fleuret,${ }^{9, b}$ M. Fontana, ${ }^{44}$ F. Fontanelli, ${ }^{21, m}$ R. Forty, ${ }^{44}$ V. Franco Lima, ${ }^{56}$ M. Frank,${ }^{44}$ C. Frei, ${ }^{44}$ J. Fu ${ }^{23, n}$ W. Funk,${ }^{44}$ E. Gabriel,${ }^{54}$ A. Gallas Torreira, ${ }^{43}$ D. Galli, ${ }^{17, d}$ S. Gallorini, ${ }^{25}$

S. Gambetta, ${ }^{54}$ Y. Gan, ${ }^{3}$ M. Gandelman, ${ }^{2}$ P. Gandini, ${ }^{23}$ Y. Gao, ${ }^{3}$ L. M. Garcia Martin, ${ }^{76}$ J. García Pardiñas, ${ }^{46}$ B. Garcia Plana, ${ }^{43}$ J. Garra Tico, ${ }^{51}$ L. Garrido, ${ }^{42}$ D. Gascon, ${ }^{42}$ C. Gaspar, ${ }^{44}$ G. Gazzoni, ${ }^{7}$ D. Gerick, ${ }^{14}$ E. Gersabeck, ${ }^{58}$ M. Gersabeck, ${ }^{58}$ T. Gershon, ${ }^{52}$ D. Gerstel, ${ }^{8}$ Ph. Ghez, ${ }^{6}$ V. Gibson, ${ }^{51}$ O. G. Girard ${ }^{45}$ P. Gironella Gironell, ${ }^{42}$ L. Giubega,${ }^{34}$ K. Gizdov, ${ }^{54}$ V. V. Gligorov, ${ }^{10}$ C. Göbel, ${ }^{65}$ D. Golubkov, ${ }^{36}$ A. Golutvin, ${ }^{57,73}$ A. Gomes, ${ }^{1,0}$ I. V. Gorelov, ${ }^{37}$ C. Gotti,${ }^{22, c}$ E. Govorkova, ${ }^{29}$ J. P. Grabowski, ${ }^{14}$ R. Graciani Diaz, ${ }^{42}$ L. A. Granado Cardoso,${ }^{44}$ E. Graugés, ${ }^{42}$ E. Graverini, ${ }^{46}$ G. Graziani, ${ }^{19}$ A. Grecu, ${ }^{34}$ R. Greim, ${ }^{29}$ P. Griffith, ${ }^{24}$ L. Grillo, ${ }^{58}$ L. Gruber, ${ }^{44}$ B. R. Gruberg Cazon,${ }^{59}$ C. Gu, ${ }^{3}$ E. Gushchin, ${ }^{38}$ A. Guth, ${ }^{11}$ Yu. Guz,${ }^{41,44}$ T. Gys, ${ }^{44}$ T. Hadavizadeh,${ }^{59}$ C. Hadjivasiliou, ${ }^{7}$ G. Haefeli, ${ }^{45}$ C. Haen, ${ }^{44}$ S. C. Haines,${ }^{51}$ B. Hamilton, ${ }^{62}$ Q. Han ${ }^{68}$ X. Han ${ }^{14}$ T. H. Hancock, ${ }^{59}$ S. Hansmann-Menzemer,${ }^{14}$ N. Harnew, ${ }^{59}$ T. Harrison,${ }^{56}$ C. Hasse, ${ }^{44}$ M. Hatch, ${ }^{44}$ J. He, ${ }^{4}$ M. Hecker, ${ }^{57}$ K. Heinicke, ${ }^{12}$ A. Heister, ${ }^{12}$ K. Hennessy, ${ }^{56}$ L. Henry, ${ }^{76}$ M. Heß,${ }^{70}$ J. Heuel, ${ }^{11}$ A. Hicheur, ${ }^{64}$ R. Hidalgo Charman, ${ }^{58}$ D. Hill, ${ }^{59}$ M. Hilton, ${ }^{58}$ P. H. Hopchev,${ }^{45}$ J. Hu, ${ }^{14}$ W. Hu, ${ }^{68}$ W. Huang, ${ }^{4}$ Z. C. Huard,${ }^{61}$

W. Hulsbergen, ${ }^{29}$ T. Humair, ${ }^{57}$ M. Hushchyn, ${ }^{74}$ D. Hutchcroft, ${ }^{56}$ D. Hynds, ${ }^{29}$ P. Ibis, ${ }^{12}$ M. Idzik,${ }^{32}$ P. Ilten, ${ }^{49}$ A. Inglessi, ${ }^{35}$ A. Inyakin, ${ }^{41}$ K. Ivshin, ${ }^{35}$ R. Jacobsson,${ }^{44}$ S. Jakobsen, ${ }^{44}$ J. Jalocha, ${ }^{59}$ E. Jans, ${ }^{29}$ B. K. Jashal, ${ }^{76}$ A. Jawahery, ${ }^{62}$ F. Jiang, ${ }^{3}$ M. John, ${ }^{59}$ D. Johnson, ${ }^{44}$ C. R. Jones,${ }^{51}$ C. Joram, ${ }^{44}$ B. Jost, ${ }^{44}$ N. Jurik, ${ }^{59}$ S. Kandybei, ${ }^{47}$ M. Karacson, ${ }^{44}$ J. M. Kariuki, ${ }^{50}$ 
S. Karodia, ${ }^{55}$ N. Kazeev, ${ }^{74}$ M. Kecke, ${ }^{14}$ F. Keizer, ${ }^{51}$ M. Kelsey, ${ }^{63}$ M. Kenzie,${ }^{51}$ T. Ketel, ${ }^{30}$ B. Khanji, ${ }^{44}$ A. Kharisova, ${ }^{75}$ C. Khurewathanakul, ${ }^{45}$ K. E. Kim, ${ }^{63}$ T. Kirn, ${ }^{11}$ V. S. Kirsebom, ${ }^{45}$ S. Klaver, ${ }^{20}$ K. Klimaszewski, ${ }^{33}$ S. Koliiev, ${ }^{48}$ M. Kolpin, ${ }^{14}$ R. Kopecna, ${ }^{14}$ P. Koppenburg, ${ }^{29}$ I. Kostiuk, ${ }^{29,48}$ O. Kot, ${ }^{48}$ S. Kotriakhova, ${ }^{35}$ M. Kozeiha, ${ }^{7}$ L. Kravchuk, ${ }^{38}$ M. Kreps, ${ }^{52}$ F. Kress, ${ }^{57}$ S. Kretzschmar, ${ }^{11}$ P. Krokovny, ${ }^{40, f}$ W. Krupa, ${ }^{32}$ W. Krzemien, ${ }^{33}$ W. Kucewicz, ${ }^{31, p}$ M. Kucharczyk ${ }^{31}$ V. Kudryavtsev, ${ }^{40, f}$ G. J. Kunde, ${ }^{78}$ A. K. Kuonen, ${ }^{45}$ T. Kvaratskheliya, ${ }^{36}$ D. Lacarrere, ${ }^{44}$ G. Lafferty, ${ }^{58}$ A. Lai, ${ }^{24}$ D. Lancierini, ${ }^{46}$ G. Lanfranchi, ${ }^{20}$ C. Langenbruch, ${ }^{11}$ T. Latham, ${ }^{52}$ C. Lazzeroni, ${ }^{49}$ R. Le Gac,${ }^{8}$ R. Lefèvre, ${ }^{7}$ A. Leflat, ${ }^{37}$ F. Lemaitre, ${ }^{44}$ O. Leroy, ${ }^{8}$ T. Lesiak, ${ }^{31}$ B. Leverington, ${ }^{14} \mathrm{H} . \mathrm{Li},{ }^{66}$ P.-R. Li ${ }^{4,9} \mathrm{X} . \mathrm{Li},{ }^{78} \mathrm{Y} . \mathrm{Li},{ }^{5} \mathrm{Z} . \mathrm{Li},{ }^{63}$ X. Liang,${ }^{63}$ T. Likhomanenko, ${ }^{72}$ R. Lindner, ${ }^{44}$ F. Lionetto, ${ }^{46}$ V. Lisovskyi, ${ }^{9}$ G. Liu, ${ }^{66}$ X. Liu, ${ }^{3}$ D. Loh, ${ }^{52}$ A. Loi, ${ }^{24}$ I. Longstaff, ${ }^{55}$ J. H. Lopes, ${ }^{2}$ G. Loustau, ${ }^{46}$ G. H. Lovell, ${ }^{51}$ D. Lucchesi, ${ }^{25, \mathrm{r}}$ M. Lucio Martinez, ${ }^{43}$ Y. Luo, ${ }^{3}$ A. Lupato, ${ }^{25}$ E. Luppi, ${ }^{18, g}$ O. Lupton, ${ }^{52}$ A. Lusiani, ${ }^{26}$ X. Lyu, ${ }^{4}$ F. Machefert, ${ }^{9}$ F. Maciuc, ${ }^{34}$ V. Macko, ${ }^{45}$ P. Mackowiak, ${ }^{12}$ S. Maddrell-Mander,${ }^{50}$ O. Maev ${ }^{35,44}$ K. Maguire, ${ }^{58}$ D. Maisuzenko, ${ }^{35}$ M. W. Majewski, ${ }^{32}$ S. Malde, ${ }^{59}$ B. Malecki, ${ }^{44}$ A. Malinin, ${ }^{72}$ T. Maltsev, ${ }^{40, f}$

H. Malygina, ${ }^{14}$ G. Manca, ${ }^{24, s}$ G. Mancinelli, ${ }^{8}$ D. Marangotto, ${ }^{23, n}$ J. Maratas, ${ }^{7, t}$ J. F. Marchand,${ }^{6}$ U. Marconi, ${ }^{17}$ C. Marin Benito, ${ }^{9}$ M. Marinangeli, ${ }^{45}$ P. Marino, ${ }^{45}$ J. Marks, ${ }^{14}$ P. J. Marshall, ${ }^{56}$ G. Martellotti ${ }^{28}$ M. Martinelli, ${ }^{44,22}$ D. Martinez Santos, ${ }^{43}$ F. Martinez Vidal, ${ }^{76}$ A. Massafferri, ${ }^{1}$ M. Materok, ${ }^{11}$ R. Matev ${ }^{44}$ A. Mathad, ${ }^{46}$ Z. Mathe, ${ }^{44}$ V. Matiunin, ${ }^{36}$ C. Matteuzzi, ${ }^{22}$ K. R. Mattioli, ${ }^{77}$ A. Mauri, ${ }^{46}$ E. Maurice, ${ }^{9, b}$ B. Maurin, ${ }^{45}$ M. McCann, ${ }^{57,44}$ A. McNab, ${ }^{58}$ R. McNulty, ${ }^{15}$ J. V. Mead, ${ }^{56}$ B. Meadows,${ }^{61}$ C. Meaux, ${ }^{8}$ N. Meinert, ${ }^{70}$ D. Melnychuk,${ }^{33}$ M. Merk, ${ }^{29}$ A. Merli, ${ }^{23, n}$ E. Michielin, ${ }^{25}$ D. A. Milanes, ${ }^{69}$ E. Millard ${ }^{52}$ M.-N. Minard, ${ }^{6}$ L. Minzoni, ${ }^{18, g}$ D. S. Mitzel, ${ }^{14}$ A. Mödden, ${ }^{12}$ A. Mogini, ${ }^{10}$ R. D. Moise, ${ }^{57}$ T. Mombächer, ${ }^{12}$ I. A. Monroy, ${ }^{69}$ S. Monteil, ${ }^{7}$ M. Morandin, ${ }^{25}$ G. Morello, ${ }^{20}$ M. J. Morello, ${ }^{26, u}$ J. Moron, ${ }^{32}$ A. B. Morris, ${ }^{8}$ R. Mountain, ${ }^{63}$ F. Muheim, ${ }^{54}$ M. Mukherjee, ${ }^{68}$ M. Mulder ${ }^{29}$ D. Müller, ${ }^{44}$ J. Müller, ${ }^{12}$ K. Müller, ${ }^{46}$ V. Müller, ${ }^{12}$ C. H. Murphy, ${ }^{59}$ D. Murray, ${ }^{58}$ P. Naik,${ }^{50}$ T. Nakada, ${ }^{45}$ R. Nandakumar, ${ }^{53}$ A. Nandi,${ }^{59}$ T. Nanut, ${ }^{45}$ I. Nasteva, ${ }^{2}$ M. Needham,${ }^{54}$ N. Neri,${ }^{23, \mathrm{n}}$ S. Neubert, ${ }^{14}$ N. Neufeld, ${ }^{44}$ R. Newcombe, ${ }^{57}$ T. D. Nguyen, ${ }^{45}$ C. Nguyen-Mau, ${ }^{45, v}$

S. Nieswand, ${ }^{11}$ R. Niet, ${ }^{12}$ N. Nikitin, ${ }^{37}$ N. S. Nolte, ${ }^{44}$ A. Oblakowska-Mucha, ${ }^{32}$ V. Obraztsov,${ }^{41}$ S. Ogilvy, ${ }^{55}$ D. P. O'Hanlon, ${ }^{17}$ R. Oldeman, ${ }^{24, s}$ C. J. G. Onderwater, ${ }^{71}$ J. D. Osborn, ${ }^{77}$ A. Ossowska, ${ }^{31}$ J. M. Otalora Goicochea, ${ }^{2}$ T. Ovsiannikova, ${ }^{36}$ P. Owen, ${ }^{46}$ A. Oyanguren, ${ }^{76}$ P. R. Pais, ${ }^{45}$ T. Pajero, ${ }^{26, u}$ A. Palano, ${ }^{16}$ M. Palutan, ${ }^{20}$ G. Panshin, ${ }^{75}$ A. Papanestis, ${ }^{53}$ M. Pappagallo, ${ }^{54}$ L. L. Pappalardo, ${ }^{18, g}$ W. Parker ${ }^{62}$ C. Parkes,${ }^{58,44}$ G. Passaleva, ${ }^{19,44}$ A. Pastore, ${ }^{16}$ M. Patel,${ }^{57}$ C. Patrignani, ${ }^{17, d}$ A. Pearce, ${ }^{44}$ A. Pellegrino, ${ }^{29}$ G. Penso, ${ }^{28}$ M. Pepe Altarelli, ${ }^{44}$ S. Perazzini,${ }^{17}$ D. Pereima,${ }^{36}$ P. Perret,${ }^{7}$ L. Pescatore,${ }^{45}$ K. Petridis, ${ }^{50}$ A. Petrolini,${ }^{21, \mathrm{~m}}$ A. Petrov ${ }^{72}$ S. Petrucci,${ }^{54}$ M. Petruzzo, ${ }^{23, \mathrm{n}}$ B. Pietrzyk, ${ }^{6}$ G. Pietrzyk,${ }^{45}$ M. Pikies,${ }^{31}$ M. Pili, ${ }^{59}$ D. Pinci, ${ }^{28}$ J. Pinzino, ${ }^{44}$ F. Pisani, ${ }^{44}$ A. Piucci, ${ }^{14}$ V. Placinta,${ }^{34}$ S. Playfer, ${ }^{54}$ J. Plews,${ }^{49}$ M. Plo Casasus, ${ }^{43}$ F. Polci, ${ }^{10}$ M. Poli Lener, ${ }^{20}$ M. Poliakova, ${ }^{63}$ A. Poluektov, ${ }^{8}$ N. Polukhina,${ }^{73, w}$ I. Polyakov, ${ }^{63}$ E. Polycarpo, ${ }^{2}$

G. J. Pomery, ${ }^{50}$ S. Ponce, ${ }^{44}$ A. Popov,${ }^{41}$ D. Popov ${ }^{49,13}$ S. Poslavskii, ${ }^{41}$ E. Price,${ }^{50}$ C. Prouve, ${ }^{43}$ V. Pugatch, ${ }^{48}$

A. Puig Navarro, ${ }^{46}$ H. Pullen, ${ }^{59}$ G. Punzi,${ }^{26, i}$ W. Qian, ${ }^{4}$ J. Qin, ${ }^{4}$ R. Quagliani, ${ }^{10}$ B. Quintana, ${ }^{7}$ N. V. Raab, ${ }^{15}$ B. Rachwal, ${ }^{32}$ J. H. Rademacker, ${ }^{50}$ M. Rama, ${ }^{26}$ M. Ramos Pernas, ${ }^{43}$ M. S. Rangel, ${ }^{2}$ F. Ratnikov, ${ }^{39,74}$ G. Raven, ${ }^{30}$ M. Ravonel Salzgeber, ${ }^{44}$ M. Reboud, ${ }^{6}$ F. Redi, ${ }^{45}$ S. Reichert, ${ }^{12}$ F. Reiss, ${ }^{10}$ C. Remon Alepuz,${ }^{76}$ Z. Ren, ${ }^{3}$ V. Renaudin, ${ }^{59}$ S. Ricciardi, ${ }^{53}$ S. Richards,${ }^{50}$

K. Rinnert, ${ }^{56}$ P. Robbe,${ }^{9}$ A. Robert, ${ }^{10}$ A. B. Rodrigues,${ }^{45}$ E. Rodrigues,${ }^{61}$ J. A. Rodriguez Lopez, ${ }^{69}$ M. Roehrken, ${ }^{44}$ S. Roiser, ${ }^{44}$ A. Rollings, ${ }^{59}$ V. Romanovskiy, ${ }^{41}$ A. Romero Vidal, ${ }^{43}$ J. D. Roth, ${ }^{77}$ M. Rotondo, ${ }^{20}$ M. S. Rudolph,${ }^{63}$ T. Ruf,${ }^{44}$ J. Ruiz Vidal, ${ }^{76}$ J. J. Saborido Silva, ${ }^{43}$ N. Sagidova, ${ }^{35}$ B. Saitta, ${ }^{24, s}$ V. Salustino Guimaraes, ${ }^{65}$ C. Sanchez Gras, ${ }^{29}$ C. Sanchez Mayordomo, ${ }^{76}$ B. Sanmartin Sedes,${ }^{43}$ R. Santacesaria, ${ }^{28}$ C. Santamarina Rios, ${ }^{43}$ M. Santimaria,${ }^{20,44}$ E. Santovetti, ${ }^{27, x}$ G. Sarpis, ${ }^{58}$ A. Sarti ${ }^{20, y}$ C. Satriano, ${ }^{28, z}$ A. Satta, ${ }^{27}$ M. Saur, ${ }^{4}$ D. Savrina,${ }^{36,37}$ S. Schael, ${ }^{11}$ M. Schellenberg, ${ }^{12}$ M. Schiller, ${ }^{55}$ H. Schindler, ${ }^{44}$ M. Schmelling, ${ }^{13}$ T. Schmelzer, ${ }^{12}$ B. Schmidt, ${ }^{44}$ O. Schneider, ${ }^{45}$ A. Schopper, ${ }^{44}$ H. F. Schreiner, ${ }^{61}$ M. Schubiger, ${ }^{45}$ S. Schulte ${ }^{45}$ M. H. Schune, ${ }^{9}$ R. Schwemmer ${ }^{44}$ B. Sciascia, ${ }^{20}$ A. Sciubba ${ }^{28, y}$ A. Semennikov, ${ }^{36}$ E. S. Sepulveda, ${ }^{10}$ A. Sergi ${ }^{49,44}$ N. Serra, ${ }^{46}$ J. Serrano, ${ }^{8}$ L. Sestini, ${ }^{25}$ A. Seuthe, ${ }^{12}$ P. Seyfert, ${ }^{44}$ M. Shapkin, ${ }^{41}$ T. Shears, ${ }^{56}$ L. Shekhtman, ${ }^{40, f}$ V. Shevchenko, ${ }^{72}$ E. Shmanin, ${ }^{73}$ B. G. Siddi, ${ }^{18}$

R. Silva Coutinho, ${ }^{46}$ L. Silva de Oliveira, ${ }^{2}$ G. Simi,${ }^{25, r}$ S. Simone, ${ }^{16, k}$ I. Skiba ${ }^{18}$ N. Skidmore, ${ }^{14}$ T. Skwarnicki, ${ }^{63}$ M. W. Slater, ${ }^{49}$ J. G. Smeaton, ${ }^{51}$ E. Smith, ${ }^{11}$ I. T. Smith, ${ }^{54}$ M. Smith,${ }^{57}$ M. Soares, ${ }^{17}$ 1. Soares Lavra, ${ }^{1}$ M. D. Sokoloff, ${ }^{61}$ F. J. P. Soler ${ }^{55}$ B. Souza De Paula, ${ }^{2}$ B. Spaan, ${ }^{12}$ E. Spadaro Norella, ${ }^{23, n}$ P. Spradlin, ${ }^{55}$ F. Stagni, ${ }^{44}$ M. Stahl, ${ }^{14}$ S. Stahl, ${ }^{44}$ P. Stefko, ${ }^{45}$ S. Stefkova, ${ }^{57}$ O. Steinkamp, ${ }^{46}$ S. Stemmle, ${ }^{14}$ O. Stenyakin, ${ }^{41}$ M. Stepanova, ${ }^{35}$ H. Stevens, ${ }^{12}$ A. Stocchi, ${ }^{9}$ S. Stone ${ }^{63}$ S. Stracka, ${ }^{26}$ M. E. Stramaglia, ${ }^{45}$ M. Straticiuc, ${ }^{34}$ U. Straumann, ${ }^{46}$ S. Strokov, ${ }^{75}$ J. Sun, ${ }^{3}$ L. Sun, ${ }^{67}$ Y. Sun, ${ }^{62}$ K. Swientek, ${ }^{32}$ A. Szabelski, ${ }^{33}$ T. Szumlak,${ }^{32}$ M. Szymanski, ${ }^{4}$ Z. Tang, ${ }^{3}$ T. Tekampe, ${ }^{12}$ G. Tellarini, ${ }^{18}$ F. Teubert, ${ }^{44}$ E. Thomas, ${ }^{44}$ M. J. Tilley, ${ }^{57}$ V. Tisserand, ${ }^{7}$ S. T'Jampens, ${ }^{6}$ M. Tobin, ${ }^{5}$ S. Tolk,${ }^{44}$ L. Tomassetti, ${ }^{18, g}$ D. Tonelli, ${ }^{26}$ D. Y. Tou, ${ }^{10}$ 
R. Tourinho Jadallah Aoude, ${ }^{1}$ E. Tournefier, ${ }^{6}$ M. Traill, ${ }^{55}$ M. T. Tran, ${ }^{45}$ A. Trisovic,${ }^{51}$ A. Tsaregorodtsev, ${ }^{8}$ G. Tuci, ${ }^{26,44, i}$ A. Tully, ${ }^{51}$ N. Tuning, ${ }^{29}$ A. Ukleja, ${ }^{33}$ A. Usachov, ${ }^{9}$ A. Ustyuzhanin,,${ }^{39,74}$ U. Uwer, ${ }^{14}$ A. Vagner, ${ }^{75}$ V. Vagnoni, ${ }^{17}$ A. Valassi, ${ }^{44}$ S. Valat ${ }^{44}$ G. Valenti, ${ }^{17}$ M. van Beuzekom, ${ }^{29}$ H. Van Hecke, ${ }^{78}$ E. van Herwijnen, ${ }^{44}$ C. B. Van Hulse, ${ }^{15}$ J. van Tilburg, ${ }^{29}$ M. van Veghel, ${ }^{29}$ A. Vasiliev, ${ }^{41}$ R. Vazquez Gomez, ${ }^{44}$ P. Vazquez Regueiro, ${ }^{43}$ C. Vázquez Sierra, ${ }^{29}$ S. Vecchi, ${ }^{18}$ J. J. Velthuis, ${ }^{50}$ M. Veltri, ${ }^{19}$,aa A. Venkateswaran, ${ }^{63}$ M. Vernet, ${ }^{7}$ M. Veronesi, ${ }^{29}$ M. Vesterinen,${ }^{52}$ J. V. Viana Barbosa, ${ }^{44}$ D. Vieira, ${ }^{4}$ M. Vieites Diaz, ${ }^{43}$ H. Viemann,${ }^{70}$ X. Vilasis-Cardona, ${ }^{42, h}$ A. Vitkovskiy, ${ }^{29}$ M. Vitti, ${ }^{51}$ V. Volkov,${ }^{37}$ A. Vollhardt,${ }^{46}$ D. Vom Bruch, ${ }^{10}$ B. Voneki, ${ }^{44}$ A. Vorobyev, ${ }^{35}$ V. Vorobyev,${ }^{40, f}$ N. Voropaev,${ }^{35}$ R. Waldi, ${ }^{70}$ J. Walsh, ${ }^{26}$ J. Wang,${ }^{5}$ M. Wang, ${ }^{3}$ Y. Wang, ${ }^{68}$ Z. Wang ${ }^{46}$ D. R. Ward ${ }^{51}$ H. M. Wark, ${ }^{56}$ N. K. Watson, ${ }^{49}$ D. Websdale, ${ }^{57}$ A. Weiden, ${ }^{46}$ C. Weisser, ${ }^{60}$ M. Whitehead, ${ }^{11}$ G. Wilkinson ${ }^{59}$ M. Wilkinson, ${ }^{63}$ I. Williams, ${ }^{51}$ M. Williams,${ }^{60}$ M. R. J. Williams, ${ }^{58}$ T. Williams, ${ }^{49}$ F. F. Wilson, ${ }^{53}$ M. Winn, ${ }^{9}$ W. Wislicki, ${ }^{33}$ M. Witek, ${ }^{31}$ G. Wormser, ${ }^{9}$ S. A. Wotton, ${ }^{51}$ K. Wyllie, ${ }^{44}$ D. Xiao, ${ }^{68}$ Y. Xie, ${ }^{68}$ H. Xing, ${ }^{66}$ A. Xu, ${ }^{3}$ M. Xu, ${ }^{68}$ Q. Xu, ${ }^{4}$ Z. Xu,${ }^{6}$ Z. Xu,${ }^{3}$ Z. Yang, ${ }^{3}$ Z. Yang, ${ }^{62}$ Y. Yao, ${ }^{63}$ L. E. Yeomans,${ }^{56}$ H. Yin, ${ }^{68}$ J. Yu,${ }^{68, b b}$ X. Yuan, ${ }^{63}$ O. Yushchenko, ${ }^{41}$ K. A. Zarebski, ${ }^{49}$ M. Zavertyaev, ${ }^{13, w}$ M. Zeng, ${ }^{3}$ D. Zhang, ${ }^{68}$ L. Zhang, ${ }^{3}$ W. C. Zhang, ${ }^{3, c c}$ Y. Zhang, ${ }^{44}$ A. Zhelezov, ${ }^{14}$ Y. Zheng, ${ }^{4}$ X. Zhu, ${ }^{3}$ V. Zhukov, ${ }^{11,37}$ J. B. Zonneveld, ${ }^{54}$ and S. Zucchelli ${ }^{17, d}$

\title{
(LHCb Collaboration)
}

\author{
${ }^{1}$ Centro Brasileiro de Pesquisas Físicas (CBPF), Rio de Janeiro, Brazil \\ ${ }^{2}$ Universidade Federal do Rio de Janeiro (UFRJ), Rio de Janeiro, Brazil \\ ${ }^{3}$ Center for High Energy Physics, Tsinghua University, Beijing, China \\ ${ }^{4}$ University of Chinese Academy of Sciences, Beijing, China \\ ${ }^{5}$ Institute Of High Energy Physics (ihep), Beijing, China \\ ${ }^{6}$ Univ. Grenoble Alpes, Univ. Savoie Mont Blanc, CNRS, IN2P3-LAPP, Annecy, France \\ ${ }^{7}$ Université Clermont Auvergne, CNRS/IN2P3, LPC, Clermont-Ferrand, France \\ ${ }^{8}$ Aix Marseille Univ, CNRS/IN2P3, CPPM, Marseille, France \\ ${ }^{9}$ LAL, Univ. Paris-Sud, CNRS/IN2P3, Université Paris-Saclay, Orsay, France \\ ${ }^{10}$ LPNHE, Sorbonne Université, Paris Diderot Sorbonne Paris Cité, CNRS/IN2P3, Paris, France \\ ${ }^{11}$ I. Physikalisches Institut, RWTH Aachen University, Aachen, Germany \\ ${ }^{12}$ Fakultät Physik, Technische Universität Dortmund, Dortmund, Germany \\ ${ }^{13}$ Max-Planck-Institut für Kernphysik (MPIK), Heidelberg, Germany \\ ${ }^{14}$ Physikalisches Institut, Ruprecht-Karls-Universität Heidelberg, Heidelberg, Germany \\ ${ }^{15}$ School of Physics, University College Dublin, Dublin, Ireland \\ ${ }^{16}$ INFN Sezione di Bari, Bari, Italy \\ ${ }^{17}$ INFN Sezione di Bologna, Bologna, Italy \\ ${ }^{18}$ INFN Sezione di Ferrara, Ferrara, Italy \\ ${ }^{19}$ INFN Sezione di Firenze, Firenze, Italy \\ ${ }^{20}$ INFN Laboratori Nazionali di Frascati, Frascati, Italy \\ ${ }^{21}$ INFN Sezione di Genova, Genova, Italy \\ ${ }^{22}$ INFN Sezione di Milano-Bicocca, Milano, Italy \\ ${ }^{23}$ INFN Sezione di Milano, Milano, Italy \\ ${ }^{24}$ INFN Sezione di Cagliari, Monserrato, Italy \\ ${ }^{25}$ INFN Sezione di Padova, Padova, Italy \\ ${ }^{26}$ INFN Sezione di Pisa, Pisa, Italy \\ ${ }^{27}$ INFN Sezione di Roma Tor Vergata, Roma, Italy \\ ${ }^{28}$ INFN Sezione di Roma La Sapienza, Roma, Italy \\ ${ }^{29}$ Nikhef National Institute for Subatomic Physics, Amsterdam, Netherlands \\ ${ }^{30}$ Nikhef National Institute for Subatomic Physics and VU University Amsterdam, Amsterdam, Netherlands \\ ${ }^{31}$ Henryk Niewodniczanski Institute of Nuclear Physics Polish Academy of Sciences, Kraków, Poland \\ ${ }^{32}$ AGH-University of Science and Technology, Faculty of Physics and Applied Computer Science, Kraków, Poland \\ ${ }^{33}$ National Center for Nuclear Research (NCBJ), Warsaw, Poland \\ ${ }^{34}$ Horia Hulubei National Institute of Physics and Nuclear Engineering, Bucharest-Magurele, Romania \\ ${ }^{35}$ Petersburg Nuclear Physics Institute NRC Kurchatov Institute (PNPI NRC KI), Gatchina, Russia \\ ${ }^{36}$ Institute of Theoretical and Experimental Physics NRC Kurchatov Institute (ITEP NRC KI), Moscow, Russia, Moscow, Russia \\ ${ }^{37}$ Institute of Nuclear Physics, Moscow State University (SINP MSU), Moscow, Russia \\ ${ }^{38}$ Institute for Nuclear Research of the Russian Academy of Sciences (INR RAS), Moscow, Russia \\ ${ }^{39}$ Yandex School of Data Analysis, Moscow, Russia \\ ${ }^{40}$ Budker Institute of Nuclear Physics (SB RAS), Novosibirsk, Russia
}


${ }^{41}$ Institute for High Energy Physics NRC Kurchatov Institute (IHEP NRC KI), Protvino, Russia, Protvino, Russia

${ }^{42}$ ICCUB, Universitat de Barcelona, Barcelona, Spain

${ }^{43}$ Instituto Galego de Física de Altas Enerxías (IGFAE), Universidade de Santiago de Compostela, Santiago de Compostela, Spain

${ }^{44}$ European Organization for Nuclear Research (CERN), Geneva, Switzerland

${ }^{45}$ Institute of Physics, Ecole Polytechnique Fédérale de Lausanne (EPFL), Lausanne, Switzerland

${ }^{46}$ Physik-Institut, Universität Zürich, Zürich, Switzerland

${ }^{47}$ NSC Kharkiv Institute of Physics and Technology (NSC KIPT), Kharkiv, Ukraine

${ }^{48}$ Institute for Nuclear Research of the National Academy of Sciences (KINR), Kyiv, Ukraine

${ }^{49}$ University of Birmingham, Birmingham, United Kingdom

${ }^{50}$ H.H. Wills Physics Laboratory, University of Bristol, Bristol, United Kingdom

${ }^{51}$ Cavendish Laboratory, University of Cambridge, Cambridge, United Kingdom

${ }^{52}$ Department of Physics, University of Warwick, Coventry, United Kingdom

${ }^{53}$ STFC Rutherford Appleton Laboratory, Didcot, United Kingdom

${ }^{54}$ School of Physics and Astronomy, University of Edinburgh, Edinburgh, United Kingdom

${ }^{55}$ School of Physics and Astronomy, University of Glasgow, Glasgow, United Kingdom

${ }^{56}$ Oliver Lodge Laboratory, University of Liverpool, Liverpool, United Kingdom

${ }^{57}$ Imperial College London, London, United Kingdom

${ }^{58}$ School of Physics and Astronomy, University of Manchester, Manchester, United Kingdom

${ }^{59}$ Department of Physics, University of Oxford, Oxford, United Kingdom

${ }^{60}$ Massachusetts Institute of Technology, Cambridge, Massachusetts, USA

${ }^{61}$ University of Cincinnati, Cincinnati, Ohio, USA

${ }^{62}$ University of Maryland, College Park, Maryland, USA

${ }^{63}$ Syracuse University, Syracuse, New York, USA

${ }^{64}$ Laboratory of Mathematical and Subatomic Physics, Constantine, Algeria

[associated with Universidade Federal do Rio de Janeiro (UFRJ), Rio de Janeiro, Brazil]

${ }^{65}$ Pontifícia Universidade Católica do Rio de Janeiro (PUC-Rio), Rio de Janeiro, Brazil

[associated with Universidade Federal do Rio de Janeiro (UFRJ), Rio de Janeiro, Brazil]

${ }^{66}$ South China Normal University, Guangzhou, China (associated with Center for High Energy Physics,

Tsinghua University, Beijing, China)

${ }^{67}$ School of Physics and Technology, Wuhan University, Wuhan, China

(associated with Center for High Energy Physics, Tsinghua University, Beijing, China)

${ }^{68}$ Institute of Particle Physics, Central China Normal University, Wuhan, Hubei, China

(associated with Center for High Energy Physics, Tsinghua University, Beijing, China)

${ }^{69}$ Departamento de Fisica, Universidad Nacional de Colombia, Bogota, Colombia (associated with LPNHE,

Sorbonne Université, Paris Diderot Sorbonne Paris Cité, CNRS/IN2P3, Paris, France)

${ }^{70}$ Institut für Physik, Universität Rostock, Rostock, Germany (associated with Physikalisches Institut, Ruprecht-Karls-Universität Heidelberg, Heidelberg, Germany)

${ }^{71}$ Van Swinderen Institute, University of Groningen, Groningen, Netherlands

(associated with Nikhef National Institute for Subatomic Physics, Amsterdam, Netherlands)

${ }^{72}$ National Research Centre Kurchatov Institute, Moscow, Russia [associated with Institute of Theoretical and Experimental Physics NRC Kurchatov Institute (ITEP NRC KI), Moscow, Russia, Moscow, Russia]

${ }^{73}$ National University of Science and Technology "MISIS", Moscow, Russia [associated with Institute of Theoretical and Experimental Physics NRC Kurchatov Institute (ITEP NRC KI), Moscow, Russia, Moscow, Russia]

${ }^{74}$ National Research University Higher School of Economics, Moscow, Russia

(associated with Yandex School of Data Analysis, Moscow, Russia)

${ }^{75}$ National Research Tomsk Polytechnic University, Tomsk, Russia [associated with Institute of Theoretical and Experimental Physics NRC Kurchatov Institute (ITEP NRC KI), Moscow, Russia, Moscow, Russia]

${ }^{76}$ Instituto de Fisica Corpuscular, Centro Mixto Universidad de Valencia-CSIC, Valencia, Spain (associated with ICCUB, Universitat de Barcelona, Barcelona, Spain)

${ }^{77}$ University of Michigan, Ann Arbor, USA (associated with Syracuse University, Syracuse, New York, USA)

${ }^{78}$ Los Alamos National Laboratory (LANL), Los Alamos, USA (associated with Syracuse University, Syracuse, New York, USA)

${ }^{\mathrm{a}}$ Deceased.

${ }^{\mathrm{b}}$ Also at Laboratoire Leprince-Ringuet, Palaiseau, France.

${ }^{\mathrm{c}}$ Also at Università di Milano Bicocca, Milano, Italy.

${ }^{\mathrm{d}}$ Also at Università di Bologna, Bologna, Italy.

${ }^{\mathrm{e}}$ Also at Università di Modena e Reggio Emilia, Modena, Italy.

${ }^{\mathrm{f}}$ Also at Novosibirsk State University, Novosibirsk, Russia.

${ }^{\mathrm{g}}$ Also at Università di Ferrara, Ferrara, Italy.

${ }^{\mathrm{h}}$ Also at LIFAELS, La Salle, Universitat Ramon Llull, Barcelona, Spain. 


\footnotetext{
${ }^{\mathrm{i}}$ Also at Università di Pisa, Pisa, Italy.

${ }^{j}$ Also at H.H. Wills Physics Laboratory, University of Bristol, Bristol, United Kingdom.

${ }^{\mathrm{k}}$ Also at Università di Bari, Bari, Italy.

${ }^{1}$ Also at Sezione INFN di Trieste, Trieste, Italy.

${ }^{\mathrm{m}}$ Also at Università di Genova, Genova, Italy.

${ }^{\mathrm{n}}$ Also at Università degli Studi di Milano, Milano, Italy.

${ }^{\circ}$ Also at Universidade Federal do Triângulo Mineiro (UFTM), Uberaba-MG, Brazil.

${ }^{\mathrm{p}}$ Also at AGH-University of Science and Technology, Faculty of Computer Science, Electronics and Telecommunications, Kraków, Poland.

${ }^{\mathrm{q}}$ Also at Lanzhou University, Lanzhou, China.

${ }^{\mathrm{r}}$ Also at Università di Padova, Padova, Italy.

${ }^{\mathrm{s}}$ Also at Università di Cagliari, Cagliari, Italy.

${ }^{t}$ Also at MSU-Iligan Institute of Technology (MSU-IIT), Iligan, Philippines.

"Also at Scuola Normale Superiore, Pisa, Italy.

${ }^{v}$ Also at Hanoi University of Science, Hanoi, Vietnam.

${ }^{\mathrm{w}}$ Also at P.N. Lebedev Physical Institute, Russian Academy of Science (LPI RAS), Moscow, Russia.

${ }^{\mathrm{x}}$ Also at Università di Roma Tor Vergata, Roma, Italy.

${ }^{\mathrm{y}}$ Also at Università di Roma La Sapienza, Roma, Italy.

${ }^{\mathrm{z}}$ Also at Università della Basilicata, Potenza, Italy.

${ }^{\text {aa }}$ Also at Università di Urbino, Urbino, Italy.

${ }^{\mathrm{bb}}$ Also at Physics and Micro Electronic College, Hunan University, Changsha City, China.

${ }^{c c}$ Also at School of Physics and Information Technology, Shaanxi Normal University (SNNU), Xi' an, China.
} 